\title{
Quercetin postconditioning attenuates myocardial ischemia/reperfusion injury in rats through the PI3K/Akt pathway
}

\author{
Y. Wang, Z.Z. Zhang, Y. Wu, J.J. Ke, X.H. He and Y.L. Wang \\ Department of Anesthesiology, Zhongnan Hospital, Wuhan University, Wuhan, China
}

\begin{abstract}
Quercetin (Que), a plant-derived flavonoid, has multiple benefical actions on the cardiovascular system. The current study investigated whether Que postconditioning has any protective effects on myocardial ischemia/reperfusion (I/R) injury in vivo and its potential cardioprotective mechanisms. Male Sprague-Dawley rats were randomly allocated to 5 groups (20 animals/ group): sham, I/R, Que postconditioning, Que + LY294002 [a phosphatidylinositol 3-kinase (PI3K)/Akt signaling pathway inhibitor], and LY294002 + I/R. I/R was produced by 30-min coronary occlusion followed by 2-h reperfusion. At the end of reperfusion, myocardial infarct size and biochemical changes were compared. Apoptosis was evaluated by both TUNEL staining and measurement of activated caspase-3 immunoreactivity. The phosphorylation of Akt and protein expression of Bcl2 and Bax were determined by Western blotting. Que postconditioning significantly reduced infarct size and serum levels of creatine kinase and lactate dehydrogenase compared with the I/R group (all $P<0.05$ ). Apoptotic cardiomyocytes and caspase3 immunoreactivity were also suppressed in the Que postconditioning group compared with the I/R group (both $\mathrm{P}<0.05$ ). Akt phosphorylation and Bcl-2 expression increased after Que postconditioning, but Bax expression decreased. These effects were inhibited by LY294002. The data indicate that Que postconditioning can induce cardioprotection by activating the PI3K/ Akt signaling pathway and modulating the expression of Bcl-2 and Bax proteins.
\end{abstract}

Key words: Ischemia and reperfusion; Quercetin; Postconditioning; PI3K/Akt

\section{Introduction}

Acute myocardial infarction is a major cause of death and disability worldwide. Although early reperfusion is essential for myocardial salvage, it induces reperfusion injury, which reduces the benefits of myocardial reperfusion $(1,2)$. Ischemic postconditioning, defined as brief periods of ischemia and reperfusion $(\mathrm{I} / \mathrm{R})$ at the onset of reperfusion, has been shown to protect against lethal reperfusion injury in many species including humans (3). However, this strategy is not suitable for patients treated with thrombolytic agents. Thus, a more feasible pharmacological postcondtioning is needed. Many chemicals such as erythropoietin (4), adenosine (5), and hydrogen sulfide (6) are known to be cardioprotective when given at the onset of reperfusion, but none has been widely used.

Quercetin (Que; 3,3',4',5,7-pentahydroxyflavone) is an important member of the flavonoid family that is present in plants and red wine (7). It is nontoxic and has a broad range of pharmacological and biological activities including antioxidative, anticarcinogenic, vasoprotective, anti-inflammatory, antidiabetic, and antiplatelet effects (811). Several studies indicated that Que, when given before ischemia (preconditioning), protects the myocardium from $\mathrm{I} / \mathrm{R}$ injury through its antioxidant and antiinflammatory activities $(12,13)$. Recently, Bartekova et al. (14) reported a protective role for Que, when administered during reperfusion (postconditioning), in an isolated rat heart model. However, it is unclear whether Que postconditioning has a protective effect against myocardial I/R injury in vivo. Moreover, the molecular mechanism underlying Que-mediated cardioprotection is unknown.

Phosphoinositide 3-kinases (PI3Ks) and their downstream effector Akt are key signal transduction enzymes involved in modulating cell proliferation, survival, and apoptosis (15). Several studies have indicated that the $\mathrm{PI}$ KK/Akt signaling pathway plays a key role in cardiac protection against I/R injury $(16,17)$. It has also been shown that Que attenuates postischemic neuronal apoptosis by activating the PI3K/Akt pathway (18). However, it

Correspondence: Y.L. Wang, Department of Anesthesiology, Zhongnan Hospital, Wuhan University, No. 169 Dong-Hu Road, Wuhan 430071, China. Fax: +86-027-6781-3040. E-mail: wyl20110829@yahoo.com.cn 
is unclear whether the $\mathrm{PI} 3 \mathrm{~K} /$ Akt pathway mediates the cardioprotection of Que postconditioning.

In the present study, Que postconditioning was used as an adjuvant to attenuate myocardial $\mathrm{I} / \mathrm{R}$ injury in an in vivo rat model.

\section{Material and Methods}

\section{Animals}

Male Sprague-Dawley rats weighing $250 \pm 20 \mathrm{~g}$ were purchased from Wuhan University Animal Center (Wuhan, China). This study conformed to the Guidelines for the Care and Use of Laboratory Animals by the National Institutes of Health (NIH Publication No. 80-23), and the experimental procedures were approved by the Institutional Animal Ethics Committee of Wuhan University.

\section{In vivo myocardial I/R model}

The in vivo myocardial I/R model was modified from a previous study (17). Briefly, rats were anesthetized by an intraperitoneal (ip) injection of $40 \mathrm{mg} / \mathrm{kg} 1 \%$ pentobarbital sodium. The rats were intubated and mechanically ventilated with room air with a rodent respirator (DV2000, Shanghai Jia Peng Technology Co., Ltd., China). A left thoracotomy was carried out to expose the hearts. A 5-0 silk ligature was passed under the left anterior descending coronary artery, and a small vinyl tube was placed on top of the vessel to form a snare for reversible coronary occlussion. After $30 \mathrm{~min}$ of ischemia, the heart was reperfused for $2 \mathrm{~h}$ by releasing the snare.

\section{Experimental groups}

A total of 100 rats were randomly allocated in equal numbers $(n=20)$ to 5 groups. 1$)$ sham: rats were subjected to the surgical procedures without coronary occlusion; 2) I/R: 30-min coronary occlusion and followed by 2-h reperfusion; 3) Que postconditioning (Que): $10 \mathrm{mg} / \mathrm{kg}$ Que ip $5 \mathrm{~min}$ before reperfusion; 4) Que +LY: $10 \mathrm{mg} / \mathrm{kg}$ Que ip $5 \mathrm{~min}$ before reperfusion and $0.3 \mathrm{mg} / \mathrm{kg}$ LY294002 ip $10 \mathrm{~min}$ before reperfusion; 5) LY+I/R: $0.3 \mathrm{mg} / \mathrm{kg}$ LY294002 ip 10 min before reperfusion. Que and LY294002 (both Sigma-Aldrich, USA) were dissolved in $0.02 \%$ dimethyl sulfoxide. The doses of Que and LY294002 were administered as described previously $(19,20)$.

\section{Determination of myocardial infarct size}

After reperfusion, the ligature around the left anterior descending coronary artery was retied. Two milliliters of 2\% Evans blue (Sigma-Aldrich) was given intravenously to track the perfused region and the area at risk (AAR). Tissue was collected from the right ventricle and atrium. The left ventricle (LV) was removed, cut into 3-mm transverse slices and incubated in $1 \%$ tetrazolium chloride (Sigma-Aldrich) solution for $20 \mathrm{~min}$ to identify the infarct area. The weight of the infarct area (white), AAR (red), and nonischemic zones of the LV (blue) was measured. Infarct size is reported as a percentage of the AAR mass.

\section{Measurement of serum creatine kinase (CK) and lactate dehydrogenase (LDH)}

At the end of reperfusion, blood samples were collected and centrifuged at $800 \mathrm{~g}$ for $10 \mathrm{~min}$. Serum CK and LDH levels were measured by a colorimetric method using commercial kits (Nanjing Jiancheng Bioengineering Institute, China) according to the manufacturer's protocols. The results are reported as U/L.

\section{Apoptotic assessment}

Terminal dUTP nick end-labeling (TUNEL) and caspase- 3 activity detection were used to assess apoptosis (21). To examine cardiac myocyte apoptosis, samples of tissue from ischemic zones were fixed in $4 \%$ paraformaldehyde, embedded in paraffin, and cut into 5- $\mu \mathrm{m}$ transverse sections. The TUNEL assay was carried out using an apoptosis detection kit (Roche Applied Science, Germany) according to the manufacturer's protocols. By light microscopy, TUNEL-positive myocardial cells had brown-staining nuclei. Five visual fields were selected randomly from each block, and at least 100 cells per field were counted at $200 \times$. The apoptotic index was calculated (apoptotic cells/total cells $\times 100 \%$ ) from a total of 25 fields per sample.

To examine caspase- 3 activity in ischemic zones, immunohistochemistry was conducted using antibody against cleaved caspase-3 (Cell Signaling Technology, USA) as described previously. Tissue sections were prepared as described earlier. Slides were viewed under a light microscope at $400 \times$ magnification (Olympus BX50 Microphotographic System, Japan). Three tissue sections were selected randomly for each animal. Immunohistochemical staining intensity was measured as mean optical density using HIPAS-2000 image-analysis software (Qianli Technical Imaging, China).

\section{Western blot analysis}

At the end of reperfusion, protein extracts were prepared as previously described (22). Briefly, cardiac samples were homogenized in lysis buffer containing $50 \mathrm{mM}$ Tris- $\mathrm{HCl}, \mathrm{pH} 7.4,150 \mathrm{mM} \mathrm{NaCl}, 5 \mathrm{mM}$ EDTA, $1 \mathrm{mM}$ dithiothreitol, $1 \%$ Triton $\mathrm{X}-100$, and $1 \%$ protease inhibitor cocktail. The homogenates were centrifuged at $12,000 \mathrm{~g}$ for $15 \mathrm{~min}$, and the supernatants were collected for Western blotting. Protein concentration was determined by a bicinchoninic acid protein assay (Beyotime Biotechnology, Inc., China), and $50-\mu \mathrm{g}$ protein homogenate samples were separated on $15 \%$ SDS-polyacrylamide gels, transferred to nitrocellulose membranes (BioRad, USA), and blocked with $5 \%$ nonfat dry milk prepared in Tris-buffered saline containing $0.05 \%$ Tween 20 (TBST). The membranes were then incubated overnight at $4{ }^{\circ} \mathrm{C}$ with 
antibodies against phospho-Akt (Ser473), Akt, Bcl-2, or Bax (Cell Signaling Technology). After 4 washes in TBST, the membranes were incubated with a secondary antibody (Santa Cruz Biotechnology, Inc., USA) for $2 \mathrm{~h}$ at room temperature. Signals were detected with an enhanced chemiluminescence kit (Beyotime Biotechnology).

\section{Statistical analysis}

Data are reported as means $\pm S D$. Differences between groups were analyzed by one-way ANOVA followed by the Bonferroni post hoc test. $\mathrm{P}<0.05$ was considered to be statistically significant. Data analyses were performed with the SPSS 13.0 software (SPSS, Inc., USA).

\section{Results}

\section{Effects of Que postconditioning on I/R-induced infarct size}

The ratio of AAR to LV mass was not significantly different among the groups, suggesting a comparable degree of existing ischemic impairment (Figure 1A). The infarct size following $\mathrm{I} / \mathrm{R}$ was $50 \pm 4 \%$ in the $\mathrm{I} / \mathrm{R}$ group. Que postconditioning significantly reduced infarct size compared with the I/R group ( $27 \pm 4$ vs $50 \pm 4 \%, \mathrm{P}<0.05)$. LY294002 administration eliminated the Que-mediated reduction in infarct size $(49 \pm 4$ vs $27 \pm 4 \%, \mathrm{P}<0.05)$. Administration of LY294002 alone before reperfusion had no effect on $\mathrm{I} / \mathrm{R}$-induced infarct size $(47 \pm 3$ vs $50 \pm 5 \%$, $\mathrm{P}>0.05$; Figure 1B).

Serum CK and LDH were determined as another indicator of myocardial insult induced by I/R. Serum levels of both CK $(2679.5 \pm 194.3$ vs $1141.5 \pm 118.7 \mathrm{U} / \mathrm{L}$, $\mathrm{P}<0.05)$ and $\operatorname{LDH}(2618 \pm 197.7$ vs $901 \pm 98.7 \mathrm{U} / \mathrm{L}$, $\mathrm{P}<0.05)$ were significantly elevated in the $\mathrm{I} / \mathrm{R}$ group compared with those in the sham group. Que postconditioning reduced the levels of CK $(1642.9 \pm 194.3$ vs $2679.5 \pm 194.3 \mathrm{U} / \mathrm{L}, \mathrm{P}<0.05)$ and $\mathrm{LDH}(1273.6 \pm 176.5$ vs $2618 \pm 197.7 \mathrm{U} / \mathrm{L}, \mathrm{P}<0.05)$ compared to the I/R group. LY294002 administration eliminated the effects of Que postconditioning on CK (2675.3 \pm 202.5 vs
$1642.9 \pm 194.3 \mathrm{U} / \mathrm{L}, \mathrm{P}<0.05)$ and $\mathrm{LDH}(2523.6 \pm 198.8$ vs $1273.6 \pm 176.5 \mathrm{U} / \mathrm{L}, \mathrm{P}<0.05)$. Consistently, administration of LY294002 alone before reperfusion had no effect on $\mathrm{I} / \mathrm{R}$-induced $\mathrm{CK}$ and $\mathrm{LDH}$ release (Figure $1 \mathrm{C}$ and $\mathrm{D}$ ).

\section{Effects of Que postconditioning on I/R-induced apoptosis}

The percentage of TUNEL-positive myocardial cells following I/R was $40.2 \pm 3.5 \%$ in the I/R group. Que postconditioning significantly decreased the percentage of TUNEL-positive myocardial cells compared to the I/R group (20.6 \pm 3.4 vs $40.2 \pm 3.5 \%, P<0.05)$. LY294002 administration eliminated Que-mediated decrease in the percentage of TUNEL-positive cells $(39.2 \pm 2.9 \mathrm{vs}$ $20.6 \pm 3.4 \%, P<0.05)$. Administration of LY294002 alone before reperfusion had no effect on $\mathrm{I} / \mathrm{R}$-induced cell apoptosis (Figure 2).

To further determine the occurrence of apoptotic cell death, we measured caspase- 3 activity by immunohistochemistry. As shown in Figure 3, caspase-3 immunoreactivity was significantly increased in the $I / R$ group compared to that in the sham group $(70 \pm 3.8$ vs $12 \pm 2.5$, $\mathrm{P}<0.05)$. Que postconditioning reduced caspase-3 immunoreactivity compared to the I/R group (23.8 \pm 3.0 vs $70 \pm 3.8, \mathrm{P}<0.05)$. LY294002 administration eliminated Que-mediated reduction in caspase-3 immunoreactivity $(66.3 \pm 4.8$ vs $23.8 \pm 3.0, P<0.05)$. Administration of LY294002 alone before reperfusion had no effect on I/R-induced caspase-3 activation.

\section{Effects of Que postconditioning on Bcl-2 and Bax expression in I/R-induced hearts}

As shown in Figure 4, a significant decrease in Bcl-2 expression and an increase in Bax expression occurred following $\mathrm{I} / \mathrm{R}$ injury (both $\mathrm{P}<0.05$ ). Que postconditioning significantly increased Bcl-2 and decreased Bax expression compared with that observed in the I/R group (both $P<0.05)$. However, LY294002 abolished the effects of Que postconditioning on Bcl-2 and Bax expression (both $\mathrm{P}<0.05)$.
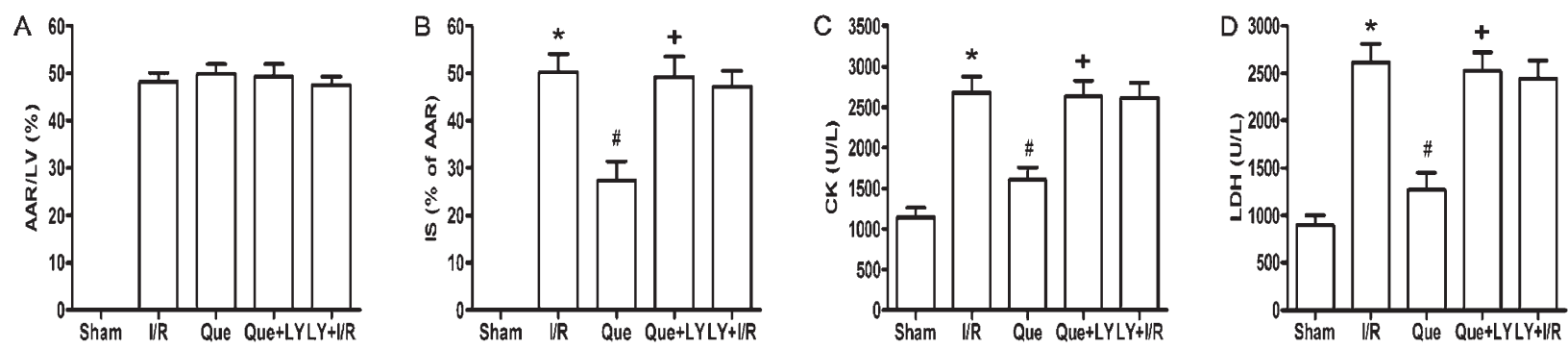

Figure 1. Effect of Que postconditioning on $\mathrm{I} / \mathrm{R}$-induced myocardial injury. $A, \mathrm{AAR}$ is reported as mean $\pm \mathrm{SD}$ percentage of the $\mathrm{LV}$ mass $(n=5) . B$, Infarct size is reported as mean \pm SD percentage of the AAR mass $(n=5)$. C, Effect of Que postconditioning on serum levels of CK $(n=8)$. D, Effect of Que postconditioning on serum levels of LDH $(n=8)$. Que: quercetin; I/R: ischemia/reperfusion; LY: LY294002; IS: infarct size; AAR: area at risk; LV: left ventricle; CK: creatine kinase; LDH: lactate dehydrogenase. ${ }^{*}<0.05$ vs sham group, ${ }^{\#} \mathrm{P}<0.05$ vs $\mathrm{l} / \mathrm{R}$ group, ${ }^{+} \mathrm{P}<0.05$ vs Que group (one-way ANOVA followed by the Bonferroni test). 

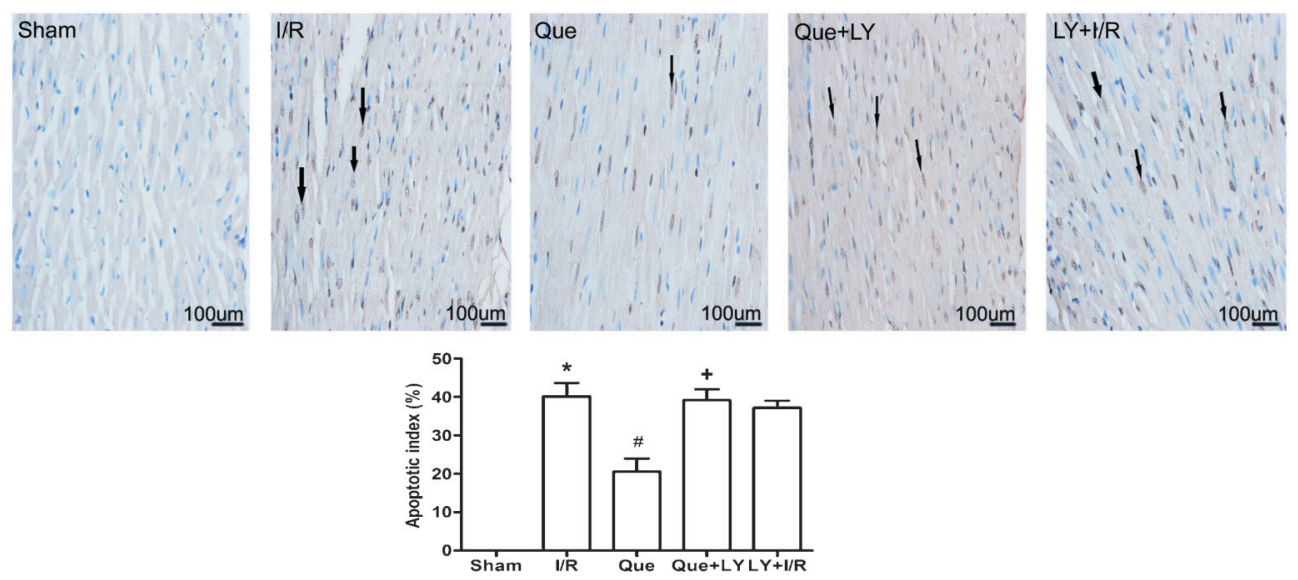

Figure 2. Effect of Que postconditioning on I/R-induced cardiomyocyte apoptosis determined by TUNEL $(n=5)$. Arrows indicate TUNEL-positive cells. Data are reported as means \pm SD. Que: quercetin; I/R: ischemia/reperfusion; LY: LY294002; TUNEL: terminal dUTP nick end-labeling. ${ }^{*} \mathrm{P}<0.05$ vs sham group, ${ }^{\#} \mathrm{P}<0.05$ vs $\mathrm{I} / \mathrm{R}$ group, ${ }^{+} \mathrm{P}<0.05$ vs Que group (one-way ANOVA followed by the Bonferroni test).

\section{Effects of Que postconditioning on Akt phosphorylation in $\mathrm{I} / \mathrm{R}$-induced hearts}

As shown in Figure 5, the ratio of P-Akt/Akt expression was significantly increased in $\mathrm{I} / \mathrm{R}$ and Que postconditioning groups compared to the sham group $(P<0.05)$. LY294002 administration inhibited the phosphorylation of Akt in Que-treated hearts $(P<0.05)$.

\section{Discussion}

There are two major findings in the current study. First, we confirmed that Que postconditioning significantly reduced infarct size and decreased cardiomyocyte apoptosis following myocardial $\mathrm{I} / \mathrm{R}$ injury in rats in vivo. Second, we confirmed that the cardioprotective effects of
Que postconditioning involved activation of the PI3K/Akt pathway by using a PI3K/Akt signaling pathway inhibitor (LY294002), which could abolish the cardioprotective effects of Que postconditioning.

The onset of myocardial ischemia is usually unpredictable clinically. Therefore, agents that confer cardioprotection at the onset of reperfusion after myocardial ischemia provide a promising approach to attenuate myocardial I/R injury. Que is a member of the flavonoid family and is widely present in the plant kingdom (7). Que manifests a wide range of beneficial biological activites and can mediate cardioprotective effects $(7,23)$. For example, an increased intake of Que has been suggested to reduce risk of cardiovascular diseases (23). Previous studies have also indicated that Que could reduce infarct
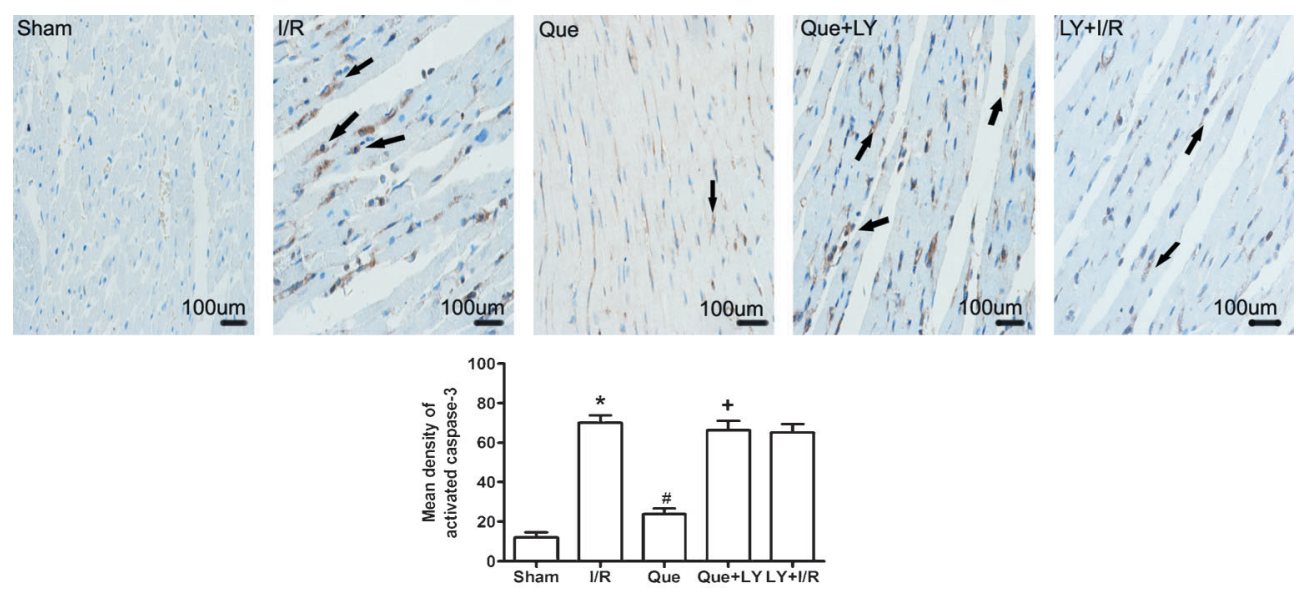

Figure 3. Immunohistochemical staining for activated caspase-3 in the myocardium after I/R injury. Arrows indicate the positive expression of activated caspase-3. Data are reported as means \pm SD. Que: quercetin; I/R: ischemia/reperfusion; LY: LY294002. ${ }^{*} \mathrm{P}<0.05$ vs sham group, ${ }^{\#} \mathrm{P}<0.05$ vs $\mathrm{I} / \mathrm{R}$ group, ${ }^{+} \mathrm{P}<0.05$ vs Que group (one-way ANOVA followed by the Bonferroni test). 

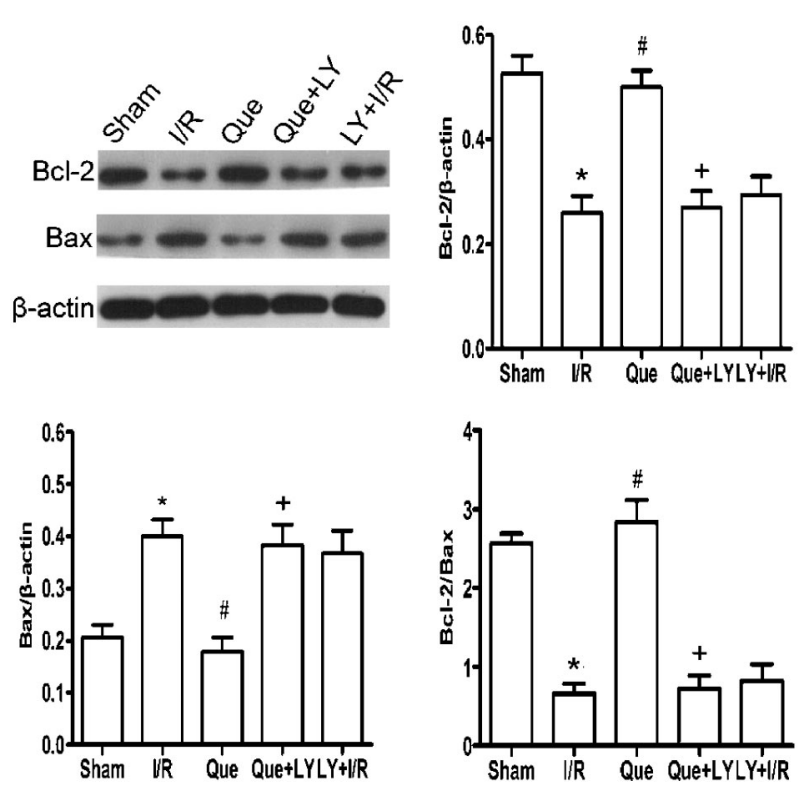

Figure 4. Effect of Que postconditioning on Bcl-2 and Bax expression after myocardial I/R injury $(n=5)$. Data are reported as means $\pm S D$. Que: quercetin; I/R: ischemia/reperfusion; LY: LY294002. ${ }^{*} \mathrm{P}<0.05$ vs sham group, ${ }^{\#} \mathrm{P}<0.05$ vs $\mathrm{I} / \mathrm{R}$ group, ${ }^{+} \mathrm{P}<0.05$ vs Que group (one-way ANOVA followed by the Bonferroni test).

size and improve functional recovery in an acute myocardial $\mathrm{I} / \mathrm{R}$ model when given prior to ischemia $(24,25)$. Similar results were observed in a myocardial infarction model induced by infusion of isoproterenol $(26,27)$. Recently, Bartekova et al. (14) described the protective effects of Que postconditioning in an isolated rat heart model of I/R injury. Our data are consistent with that study (14), and we further demonstrated that Que postconditioning exerted remarkable cardioprotective effects against myocardial $\mathrm{I} / \mathrm{R}$ injury in an in vivo rat
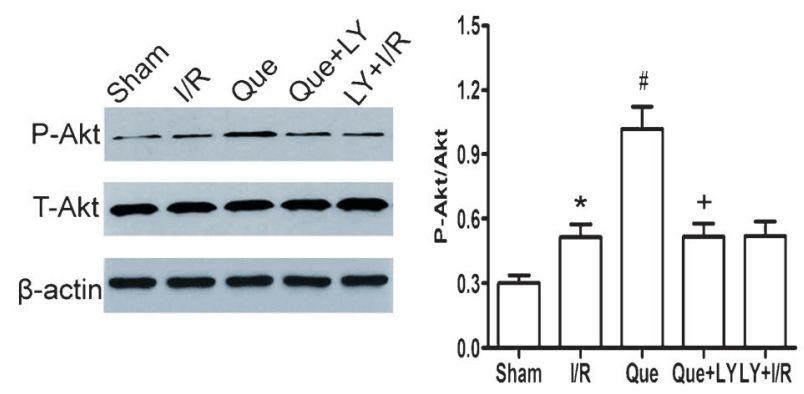

Figure 5. Effect of Que postconditioning on P-Akt expression during myocardial $I / R(n=5)$. Data are reported as means $\pm S D$. Que: quercetin; I/R: ischemia/reperfusion; LY: LY294002; P-Akt: phospho-Akt; T-Akt: total-Akt. * $\mathrm{P}<0.05$ vs sham group, ${ }^{\#} \mathrm{P}<0.05$ vs I/R group, ${ }^{+} \mathrm{P}<0.05$ vs Que group (one-way ANOVA followed by the Bonferroni test). model showing diminished infarct size and reduced CK and $\mathrm{LDH}$ release.

It is well documented that apoptosis is dominant in the pathogenesis of myocardial I/R injury. Suppression of apoptosis could reduce loss of cardiomyocytes and limit myocardial damage caused by $\mathrm{l} / \mathrm{R}(28)$. Ischemic postconditioning, which consists of brief periods of $\mathrm{I} / \mathrm{R}$ at the onset of reperfusion, has been shown to inhibit cardiomyocyte apoptosis and attenuate myocardial I/R injury (29). Similar to ischemic postconditioning, the results from TUNEL staining and immunohistochemisty for activated caspase-3 in our current study showed that Que postconditioning could significantly decrease cardiomyocyte apoptosis after I/R injury. These results suggest that antiapoptosis might be one of the major mechanisms underlying the cardioprotective effects of Que postconditioning.

Bcl-2 family members regulate apoptosis by modulating mitochondrial membrane permeability (30). The antiapoptotic protein $\mathrm{Bcl}-2$ is located in the mitochondrial wall and prevents mitochondrial release of cytochrome $\mathrm{c}$, while the proapoptotic protein Bax resides in the cytosol but can be translocated to mitochondria to induce cytochrome c release. Therefore, the ratio of the $\mathrm{Bcl}-2 /$ Bax protein is a key factor in determining cell apoptosis or survival after apoptotic stimuli (31). Experimental studies have demonstrated that ischemic postconditioning reduces apoptosis by modulating $\mathrm{Bcl}-2$ and/or $\mathrm{Bax}$ expression that prevents cardiomyocyte apoptosis following myocardial I/R (29). In the current study, we found that Que postconditioning significantly decreased Bax expression and increased $\mathrm{Bcl}-2$ expression and the ratio of $\mathrm{Bcl}-$ 2/Bax. These results indicate that antiapoptosis of Que postconditioning might be related to changes in the expression of $\mathrm{Bcl}-2$ and Bax.

It is not clear how Que modulates Bcl-2 and Bax expression during myocardial $\mathrm{I} / \mathrm{R}$ in vivo. The antioxidant action of Que may contribute to this modulation (32), since many antioxidants affect the expression of $\mathrm{Bcl}-2$ family proteins during myocardial I/R $(33,34)$. Alternatively, activation of PI3K/Akt may be responsible for the changes in Bcl-2 and Bax expression (35). PI3K/ Akt is an important component of the intracellular signaling pathway regulating cell growth, survival and apoptosis. Activating PI3K/Akt at the time of reperfusion has been demonstrated to provide protection against myocardial I/R injury $(4,18,36,37)$. Tsang et al. (36) showed that ischemic postconditioning increased phosphorylation of Akt and inhibition of Akt activity partly abolished the cardioprotective effects of ischemic postconditioning. Many cardioprotectants, including erythropoietin (4), sulfentanil (17), and KB-R7943 (37), confer their cardioprotective effects through the PI3K/Akt pathway. In this study, Que postconditioning significantly increased phosphorylation of Akt and reduced cardiomyocyte apoptosis by regulating the $\mathrm{Bcl}-2$ family. The cardioprotective effects of Que postconditioning were 
blocked by the PI3K/Akt signaling pathway inhibitor LY294002. These data indicate that Que postcondtioning induces cardioprotection through the PI3K/Akt signaling pathway. It has been shown that previously activated Akt could inhibit expression of proapoptotic proteins such as Bad, Bax, and caspase-9 (15), but maintain high levels of antiapoptotic protein $\mathrm{Bcl}-2$ (38). Akt has also been suggested to increase the production of nitric oxide (39). The protective roles of nitric oxide during ischemic postconditioning and pharmacological postconditioning have already been confirmed $(37,38)$. Additionally, signaling through the PI3K/Akt pathway could activate PKC, which plays a key role in cardiac protection against myocardial I/R injury (40). Therefore, it appears that the $\mathrm{PI}$ KK/Akt pathway contributes to the recruitment of

\section{References}

1. Yellon DM, Hausenloy DJ. Myocardial reperfusion injury. NEngl J Med 2007; 357: 1121-1135, doi: 10.1056/NEJMra071667.

2. Hausenloy DJ, Yellon DM. Myocardial ischemia-reperfusion injury: a neglected therapeutic target. J Clin Invest 2013; 123: 92-100, doi: $10.1172 / \mathrm{JCl} 62874$.

3. Penna C, Mancardi D, Raimondo S, Geuna S, Pagliaro P. The paradigm of postconditioning to protect the heart. J Cell Mol Med 2008; 12: 435-458, doi: 10.1111/j.1582-4934.2007. 00210.x

4. Tamareille S, Ghaboura N, Treguer F, Khachman D, Croue $A$, Henrion D, et al. Myocardial reperfusion injury management: erythropoietin compared with postconditioning. Am J Physiol Heart Circ Physiol 2009; 297: H2035-H2043, doi: 10.1152/ajpheart.00472.2009.

5. Ke JJ, Yu FX, Rao Y, Wang YL. Adenosine postconditioning protects against myocardial ischemia-reperfusion injury though modulate production of TNF-alpha and prevents activation of transcription factor NF-kappaB. Mol Biol Rep 2011; 38: 531-538, doi: 10.1007/s11033-010-0137-8.

6. Ji Y, Pang QF, Xu G, Wang L, Wang JK, Zeng YM. Exogenous hydrogen sulfide postconditioning protects isolated rat hearts against ischemia-reperfusion injury. Eur J Pharmacol 2008; 587: 1-7, doi: 10.1016/j.ejphar. 2008.03.044.

7. Smith AJ, Kavuru P, Wojtas L, Zaworotko MJ, Shytle RD. Cocrystals of quercetin with improved solubility and oral bioavailability. Mol Pharm 2011; 8: 1867-1876, doi: 10.1021/ $\mathrm{mp200209j.}$

8. Staedler D, Idrizi E, Kenzaoui BH, Juillerat-Jeanneret L. Drug combinations with quercetin: doxorubicin plus quercetin in human breast cancer cells. Cancer Chemother Pharmacol 2011; 68: 1161-1172, doi: 10.1007/s00280011-1596-x

9. Cheng S, Gao N, Zhang Z, Chen G, Budhraja A, Ke Z, et al. Quercetin induces tumor-selective apoptosis through downregulation of Mcl-1 and activation of Bax. Clin Cancer Res 2010; 16: 5679-5691, doi: 10.1158/1078-0432.CCR-10-1565.

10. Noori-Daloii MR, Momeny M, Yousefi M, Shirazi FG, Yaseri $\mathrm{M}$, Motamed N, et al. Multifaceted preventive effects of single agent quercetin on a human prostate adenocarcinoma cell line (PC-3): implications for nutritional transcriptomics and various endogenous cardioprotective signals to attenuate myocardial I/R injury. Furture research is needed to identify other signaling elements involved in Que-induced cardioprotection against myocardial I/R injury.

In the present study, we demonstrated that Que postconditioning attenuates myocardial $\mathrm{I} / \mathrm{R}$ injury in rats via activating the PI3K/Akt pathway and modulating $\mathrm{Bcl}-2$ and Bax expression.

\section{Acknowledgments}

Research supported by the National Natural Science Foundation of China (\#3872426 and \#303164136) and the Fundamental Research Funds for the Central Universities (\#20103030101000205). multi-target therapy. Med Oncol 2011; 28: 1395-1404, doi: 10.1007/s12032-010-9603-3.

11. Dodson HC, Lyda TA, Chambers JW, Morris MT, Christensen KA, Morris JC. Quercetin, a fluorescent bioflavanoid, inhibits Trypanosoma brucei hexokinase 1. Exp Parasitol 2011; 127: 423-428, doi: 10.1016/j.exppara.2010.10.011.

12. Jin HB, Yang YB, Song YL, Zhang YC, Li YR. Protective roles of quercetin in acute myocardial ischemia and reperfusion injury in rats. Mol Biol Rep 2012; 39: 1100511009, doi: 10.1007/s11033-012-2002-4.

13. Wan LL, Xia J, Ye D, Liu J, Chen J, Wang G. Effects of quercetin on gene and protein expression of NOX and NOS after myocardial ischemia and reperfusion in rabbit. Cardiovasc Ther 2009; 27: 28-33, doi: 10.1111/j.1755-5922. 2009.00071.x.

14. Bartekova M, Carnicka S, Pancza D, Ondrejcakova M, Breier A, Ravingerova T. Acute treatment with polyphenol quercetin improves postischemic recovery of isolated perfused rat hearts after global ischemia. Can $J$ Physiol Pharmacol 2010; 88: 465-471, doi: 10.1139/Y10-025.

15. Cantley LC. The phosphoinositide 3-kinase pathway. Science 2002; 296: 1655-1657, doi: 10.1126/science.296. 5573.1655

16. Xu X, Cao Z, Cao B, Li J, Guo L, Que L, et al. Carbamylated erythropoietin protects the myocardium from acute ischemia/reperfusion injury through a PI3K/Akt-dependent mechanism. Surgery 2009; 146: 506-514, doi: 10.1016/ j.surg.2009.03.022.

17. Wu QL, Shen T, Ma H, Wang JK. Sufentanil postconditioning protects the myocardium from ischemia-reperfusion via PI3K/Akt-GSK-3beta pathway. J Surg Res 2012; 178: 563570, doi: 10.1016/j.jss.2012.05.081.

18. Yao RQ, Qi DS, Yu HL, Liu J, Yang LH, Wu XX. Quercetin attenuates cell apoptosis in focal cerebral ischemia rat brain via activation of BDNF-TrkB-PI3K/Akt signaling pathway. Neurochem Res 2012; 37: 2777-2786, doi: 10.1007/s11064012-0871-5.

19. Annapurna A, Reddy CS, Akondi RB, Rao SR. Cardioprotective actions of two bioflavonoids, quercetin and rutin, in experimental myocardial infarction in both normal and streptozotocin-induced type I diabetic rats. $J$ 
Pharm Pharmacol 2009; 61: 1365-1374, doi: 10.1211/ jpp.61.10.0014.

20. Zhang JY, Chen ZW, Yao H. Protective effect of urantide against ischemia-reperfusion injury via protein kinase $C$ and phosphatidylinositol 3'-kinase - Akt pathway. Can J Physiol Pharmacol 2012; 90: 637-645, doi: 10.1139/y2012-048.

21. Ha T, Hua F, Liu X, Ma J, McMullen JR, Shioi T, et al. Lipopolysaccharide-induced myocardial protection against ischaemia/reperfusion injury is mediated through a $\mathrm{PI} 3 \mathrm{~K} /$ Akt-dependent mechanism. Cardiovasc Res 2008; 78: 546553, doi: $10.1093 / \mathrm{cvr} / \mathrm{cvn} 037$.

22. Zhang S, Li H, Yang SJ. Tribulosin protects rat hearts from ischemia/reperfusion injury. Acta Pharmacol Sin 2010; 31: 671-678, doi: 10.1038/aps.2010.45.

23. Perez-Vizcaino F, Duarte J. Flavonols and cardiovascular disease. Mol Aspects Med 2010; 31: 478-494, doi: 10.1016/ j.mam.2010.09.002.

24. Punithavathi VR, Prince PS. Pretreatment with a combination of quercetin and alpha-tocopherol ameliorates adenosine triphosphatases and lysosomal enzymes in myocardial infarcted rats. Life Sci 2010; 86: 178-184, doi: 10.1016/ j.lfs.2009.11.021.

25. Brookes PS, Digerness SB, Parks DA, Darley-Usmar V. Mitochondrial function in response to cardiac ischemiareperfusion after oral treatment with quercetin. Free Radic Biol Med 2002; 32: 1220-1228, doi: 10.1016/S08915849(02)00839-0.

26. Punithavathi VR, Stanely Mainzen Prince P. The cardioprotective effects of a combination of quercetin and alphatocopherol on isoproterenol-induced myocardial infarcted rats. J Biochem Mol Toxicol 2011; 25: 28-40, doi: 10.1002/ jbt.20357.

27. Punithavathi VR, Prince PS. Combined effects of quercetin and alpha-tocopherol on lipids and glycoprotein components in isoproterenol induced myocardial infarcted Wistar rats. Chem Biol Interact 2009; 181: 322-327, doi: 10.1016/ j.cbi.2009.07.002.

28. Song JQ, Teng X, Cai Y, Tang CS, Qi YF. Activation of Akt/ GSK-3beta signaling pathway is involved in intermedin(153) protection against myocardial apoptosis induced by ischemia/reperfusion. Apoptosis 2009; 14: 1061-1069, doi: 10.1007/s10495-009-0382-2.

29. Sun H, Guo T, Liu L, Yu Z, Xu W, Chen W, et al. Ischemic postconditioning inhibits apoptosis after acute myocardial infarction in pigs. Heart Surg Forum 2010; 13: E305-E310, doi: 10.1532/HSF98.20101013.

30. Valen $\mathrm{G}$. The basic biology of apoptosis and its implications for cardiac function and viability. Ann Thorac Surg 2003; 75 : S656-S660, doi: 10.1016/S0003-4975(02)04687-8.

31. Zhao ZQ, Nakamura M, Wang NP, Wilcox JN, Shearer S, Ronson RS, et al. Reperfusion induces myocardial apoptotic cell death. Cardiovasc Res 2000; 45: 651-660, doi: 10.1016/ S0008-6363(99)00354-5.

32. Park C, So HS, Shin $\mathrm{CH}$, Baek SH, Moon BS, Shin SH, et al. Quercetin protects the hydrogen peroxide-induced apoptosis via inhibition of mitochondrial dysfunction in $\mathrm{H} 9 \mathrm{c} 2$ cardiomyoblast cells. Biochem Pharmacol 2003; 66: 12871295, doi: 10.1016/S0006-2952(03)00478-7.

33. Jeong JJ, Ha YM, Jin YC, Lee EJ, Kim JS, Kim HJ, et al. Rutin from Lonicera japonica inhibits myocardial ischemia/ reperfusion-induced apoptosis in vivo and protects $\mathrm{H} 9 \mathrm{c} 2$ cells against hydrogen peroxide-mediated injury via ERK $1 / 2$ and PI3K/Akt signals in vitro. Food Chem Toxicol 2009; 47: 1569-1576, doi: 10.1016/j.fct.2009.03.044.

34. Luan HF, Zhao ZB, Zhao QH, Zhu P, Xiu MY, Ji Y. Hydrogen sulfide postconditioning protects isolated rat hearts against ischemia and reperfusion injury mediated by the JAK2/STAT3 survival pathway. Braz J Med Biol Res 2012; 45: 898-905.

35. Vecchione C, Patrucco E, Marino G, Barberis L, Poulet R, Aretini $A$, et al. Protection from angiotensin II-mediated vasculotoxic and hypertensive response in mice lacking PI3Kgamma. J Exp Med 2005; 201: 1217-1228, doi: 10.1084/jem.20040995.

36. Tsang A, Hausenloy DJ, Mocanu MM, Yellon DM. Postconditioning: a form of "modified reperfusion" protects the myocardium by activating the phosphatidylinositol 3kinase-Akt pathway. Circ Res 2004; 95: 230-232, doi: 10.1161/01.RES.0000138303.76488.fe.

37. Ren Y, Cai Y, Jia D. Comparative antiapoptotic effects of KB-R7943 and ischemic postconditioning during myocardial ischemia reperfusion. Cell Biochem Biophys 2012; 64: 137145, doi: 10.1007/s12013-012-9382-x.

38. Uchiyama T, Engelman RM, Maulik N, Das DK. Role of Akt signaling in mitochondrial survival pathway triggered by hypoxic preconditioning. Circulation 2004; 109: 3042-3049, doi: 10.1161/01.CIR.0000130647.29030.90.

39. Guo JY, Yang T, Sun XG, Zhou NY, Li FS, Long D, et al. Ischemic postconditioning attenuates liver warm ischemiareperfusion injury through Akt-eNOS-NO-HIF pathway. $J$ Biomed Sci 2011; 18: 79, doi: 10.1186/1423-0127-18-79.

40. Murphy E, Steenbergen C. Mechanisms underlying acute protection from cardiac ischemia-reperfusion injury. Physiol Rev 2008; 88: 581-609, doi: 10.1152/physrev.00024.2007. 